\title{
The Coexistence of RAS and BRAF Mutations in Metastatic Colorectal Cancer: A Case Report and Systematic Literature Review
}

\author{
Vlad-Adrian Afrăsânie ${ }^{1}$, Bogdan Gafton ${ }^{1,2}$, Mihai Vasile Marinca ${ }^{1,2}$, Teodora Alexa-Stratulat ${ }^{1,2}$, Lucian Miron ${ }^{1,2}$, Cristina \\ Rusu $^{1}$, Anca-Viorica Ivanov ${ }^{1}$, Gheorghe G Balan ${ }^{1,3}$, Adina-Emilia Croitoru ${ }^{4,5}$
}

1) Gr. T. Popa University of

Medicine and Pharmacy, Iași 2) Oncology Dept., Regiona Institute of Oncology, Iași 3) Gastroenterology Dept., Sf. Spiridon University Clinical Emergency Hospital, Iași

4) Titu Maiorescu University of Medicine, Bucharest 5) Oncology Dept., Fundeni Clinical Institute, Bucharest, Romania

Address for correspondence: Bogdan Gafton Gr. T. Popa University of Medicine and Pharmacy, Oncology Dept., Regional Institute of Oncology, Iași, Romania

gaftonbogdan@yahoo.com
Received: 18.02.2020 Accepted: 13.05.2020

\section{ABSTRACT}

Background \& Aims: The coexistence of $R A S$ and $B R A F$ mutations is extremely rare, occurring in approximately $0.05 \%$ of patients with metastatic colorectal cancer (mCRC). Starting from a case presentation, this review aims to examine the prevalence, clinical, histopathological and molecular features of tumors with concomitant mutations.

Methods: Case report and systematic review. We performed a systematic literature search in PubMed and EMBASE using the following MeSH terms: "coexistence" OR "concomitant" AND "RAS" AND "BRAF" AND "colorectal cancer" from the inception of the databases onwards.

Results: We present the case of a 53-year-old man diagnosed with metastatic rectal adenocarcinoma with both a KRAS and a BRAF mutation. The review included eleven papers reporting on atot of $30 \mathrm{mCRC}$ cases with concomitant $R A S$ and $B R A F$ mutations. The male/female ratio was $11 / 5$. The average age was 58.5 years. The tumor was located in nine cases on the right colon and in two cases in the left colon. $43.3 \%$ of subjects had liver metastases, and 6.6\% had lung metastases. Next-generation sequencing (NGS) was used in 36.6\% of cases and polymerase chain reaction (PCR) in $16.6 \%$ of cases. KRAS mutations were present in $83.3 \%$ of patients and NRAS mutations in $16.6 \%$ of patients. Survival could be assessed in 10 patients and the median was 21.1 months (about 30\% lower than the survival in the general mCRC population).

Conclusion: The results of this systematic review suggest the need to design a cohort study (either prospective or retrospective) to better characterize the patients with concomitant $R A S$ and $B R A F$ mutations and to establish the optimal treatment for this rare situation.

Key words: metastatic colorectal cancer - concomitant mutations $-R A S-B R A F$.

Abbreviations: BRAF: v-raf murine sarcoma viral oncogene homolog B1; CRC: colorectal cancer; CT: computed tomography; EGFR: epithelial growth factor receptor; FU: fluorouracil; KRAS: Kirsten rat sarcoma viral oncogene homolog; mCRC: metastatic CRC; MEK inhibitor: mitogen-activated protein kinase inhibitor; MSI: microsatellite instability; NRAS: neuroblastoma rat sarcoma viral oncogene homolog; NGS: next-generation sequencing; OS: overall survival; PCR: polymerase chain reaction; PFS: progression-free survival; RAS: rat sarcoma.

\section{INTRODUCTION}

Colorectal cancer (CRC) has a central role in cancer pathology worldwide; it is the second most common cancer in women and the third in men [1]. Over the last 20 years, significant therapeutic progress has been achieved in the metastatic stage of the disease, which has resulted in increased survival rates $[2,3]$. Currently, rat sarcoma $(R A S)$ and $\mathrm{v}$-raf murine sarcoma viral oncogene homolog $\mathrm{B} 1(B R A F)$ mutations are known to be crucial mediators on the epithelial growth factor receptor (EGFR) signaling pathway, and essential factors in colorectal carcinogenesis [4,5]. RAS mutations occur in approximately $40 \%$ of mCRC patients, while $B R A F$ mutations are present in $8-12 \%$ of cases [6]. RAS mutations are predictive factors for primary resistance to anti-EGFR biological therapies. It was shown that the addition of antiEGFR agents to standard chemotherapy regimens, such as FOLFOX (leucovorin, 5-Fluorouracil (5-FU) and oxaliplatin) or FOLFIRI (leucovorin, 5-FU and irinotecan), does not produce benefits for patients with RAS mutations [7-10]. The data is insufficient and less clear in order to draw a conclusion regarding the predictive value of $B R A F$ mutations for antiEGFR therapies [11]. It is widely accepted that RAS and $B R A F$ are mutually exclusive in almost all cases. Rarely, however, CRC 
patients may present with concomitant Kirsten rat sarcoma viral oncogene homolog (KRAS) and BRAF mutations (0.05\%). The clinical outcomes and prognostic role of concomitant mutations in CRC patients are yet to be fully understood and quantified [4].

\section{CASE REPORT}

A 53-year-old Caucasian male, accountant, presented at our hospital with severe rectal bleeding. The patient had no relevant medical or family history, did not smoke, and drank alcohol occasionally. His performance status was 1 on the ECOG (Eastern Cooperative Oncology Group) scale. Clinical examination revealed hepatomegaly. The biological analysis indicated iron deficiency anemia with a hemoglobin level of $10.6 \mathrm{~g} / \mathrm{dL}$. The colonoscopy detected a vegetative tumor located $6 \mathrm{~cm}$ from the anal verge, and histological analysis showed adenocarcinoma. Pelvic magnetic resonance imaging (MRI) identified a $67 \mathrm{~mm}$-long irregular circumferential thickening of the lower and median rectal wall at $40 \mathrm{~mm}$ from the anus, extending into the perirectal fatty area. The abdominal computed tomography (CT) scan showed an enlarged liver with multiple nodules ranging between $2 \mathrm{~mm}$ and $106 \mathrm{~mm}$ in size, suggestive for liver metastases. The case was discussed in our multidisciplinary tumor board and rectal surgery (Hartmann surgical approach) was decided for severe rectal bleeding. The pathology exam indicated pT3pN1b G2 rectal adenocarcinoma. The mutational status of the RAS and $B R A F$ genes was analyzed by polymerase chain reaction (PCR) from the surgical specimen. KRAS mutation (G12D) and BRAF mutation (position 600 of exon 15 (V600E)) were identified. Immunohistochemistry testing for microsatellite instability (MSI) did not show a MSI-high profile. Two months later, the patient was admitted in Medical Oncology Department and received first-line palliative chemotherapy with the FOLFOX-4 regimen (oxaliplatin $85 \mathrm{mg} / \mathrm{m}^{2}$ iv on day 1, 5-FU $400 \mathrm{mg} / \mathrm{m}^{2}$ iv bolus followed by $600 \mathrm{mg} / \mathrm{m}^{2}$ iv 22-hour continuous infusion on days 1 and 2, and leucovorin: $200 \mathrm{mg} / \mathrm{m}^{2}$ iv on days 1 and 2) plus bevacizumab ( $5 \mathrm{mg} / \mathrm{kg})$ on a two-week schedule. After twelve cycles, the patient developed grade 3 sensitive peripheral neuropathy. Oxaliplatin was discontinued, and treatment was continued with capecitabine $\left(1000 \mathrm{mg} / \mathrm{m}^{2}\right.$ day 1 to 14$)$ and bevacizumab $7.5 \mathrm{mg} / \mathrm{kg}$ every three weeks.

At six months after starting the chemotherapy, the disease was stable according to response evaluation criteria in solid tumors (RECIST) 1.1. Over the next six months, the patient continued on capecitabine associated with bevacizumab, but the subsequent CT examination revealed progressive disease (increased number and size of liver metastases) and a new $67 / 67 / 77 \mathrm{~mm}$ rectal lesion that invaded the anal sphincter. The chemotherapy was switched to FOLFIRI (irinotecan $180 \mathrm{mg}$ / $\mathrm{m}^{2}$ iv on day 1, 5 -FU: $400 \mathrm{mg} / \mathrm{m}^{2}$ iv bolus on day 1 , followed by $2400 \mathrm{mg} / \mathrm{m}^{2}$ iv continuous infusion for 46 hours, and leucovorin: $400 \mathrm{mg} / \mathrm{m}^{2}$ iv on day one every two weeks). Bevacizumab was interrupted due to persistent grade 3 proteinuria. We proposed palliative radiotherapy, but the patient refused. After four cycles of FOLFIRI, imaging studies revealed that the patient had progressive disease at the hepatic site. The patient died due to liver failure, 23 months after diagnosis.

\section{SYSTEMATIC REVIEW OF THE LITERATURE}

An extensive literature search was done based on a predrafted protocol, including eligibility criteria, search strategies, criteria for study selection and methods for extracting data according to the Preferred Reporting Items for Systematic Review and Meta-Analysis (PRISMA) guidelines.

\section{Search methods}

Previously published articles indexed in PubMed and EMBASE were searched using the following MeSH terms and keywords: "coexistence" OR "concomitant" AND "RAS" AND "BRAF" AND "colorectal cancer". Data was gathered from the

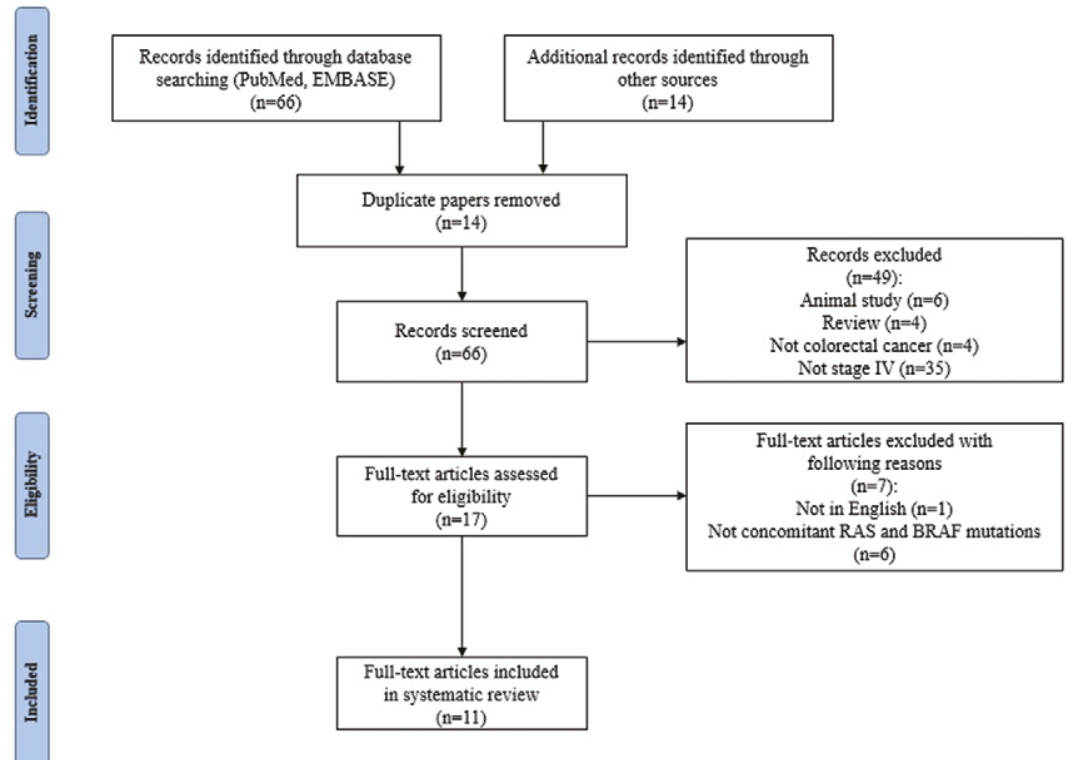

Fig. 1. PRISMA flowchart of literature search. 
inception of the databases onwards. The search was restricted to papers with full text available in English or at least with access to an abstract in English. We also included abstracts from conferences, and we evaluated the reference lists of articles to include the potentially appropriate articles. Reviews and articles that were not referring to CRC and stage IV of disease were excluded. Only reports in humans were included for this systematic review. Fig. 1 shows the flowchart of selecting the studies according to PRISMA guidelines.

\section{Eligibility criteria}

Studies were included only if the authors concluded that RAS and BRAF mutations were coexistent in one or more mCRC patients, and the results were written in English.

\section{RESULTS}

Eleven full text-articles were included, covering 30 cases in total. Gender, age, primary tumor site, location of metastases, method of determination, RAS mutation, BRAF mutation, location of mutations in codons, first-line treatment, response and survival are detailed in Supplementary Table I. However, the data collected for the studied parameters in every patient were not complete for all the 30 cases identified in the literature. The percentages are calculated to a total of 30 patients. For each parameter, we reported the percentage of missing data.

\section{Clinical characteristics}

The ratio between males and females was $11 / 5$ (36.6\%/16.6\%) and the average age of patients was 58.5 years; however, data were not available in $46.6 \%$ of cases. In nine cases (30\%) the tumor was located on the left colon and in only two cases $(6.6 \%)$ on the right colon, for the other nineteen cases $(63.3 \%)$ the tumor location was not described. Thirteen $(43.3 \%)$ patients had liver metastases, two (6.6\%) had lung metastases, and in 15 $(50 \%)$ cases, the site of the secondary lesion was not described.

\section{Molecular characteristics}

The method used to detect the mutations were: PCR in $16.6 \%$ of subjects $(n=5)$ and next-generation sequencing (NGS) in $36.6 \%(n=11)$ of cases; in $46.6 \%(n=14)$ data regarding the used technique was missing. KRAS mutations were present in $83.3 \%(n=25)$ of cases and $16.6 \%(n=5)$ of patients had neuroblastoma rat sarcoma viral oncogene homolog (NRAS) mutations. One patient had coexistent KRAS and NRAS mutation, and in one subject the type of RAS mutation was not described. KRAS mutations were most frequently found at codons G12D (26.6\%, n=8), G13D (16.7\%, n=5), G12V (10\%, $\mathrm{n}=3$ ) and other mutation types were found in every other patient and counted for 30\% $(n=9)$. NRAS mutations were identified in different codons: G12A ( $n=1)$, G12D ( $n=2)$, G13S $(\mathrm{n}=1)$, Q61Q $(\mathrm{n}=1)$. BRAF mutations were most frequently located in codons V600E $(50 \%, n=15), \operatorname{V600D}(6.6 \%, n=2)$, D594G (6.6\%, n=2), G466V (6.6\%, n=2).

\section{Management and outcome}

Information about treatment was available only for ten patients $(33.3 \%)$, in whom FOLFOX $(20 \%, n=6)$, FOLFIRI $(10 \%, \mathrm{n}=3)$ and IFL (irinotecan, leucovorin and 5-FU)
$(3.3 \%, n=1)$ were the most commonly used chemotherapy regimens. Bevacizumab was administered in association with chemotherapy only in 4 patients. The responses were mixed, and no conclusion could be drawn regarding the best therapeutic approach. Survival data was available only for ten patients, averaging 21.6 months [4, 5, 6, 11-19].

\section{DISCUSSION}

The RAS protein is a crucial factor in regulating cellular mitosis, and its expression and activity are amplified by activating mutations in its coding gene. KRAS mutations occur in approximately $40 \%$ of cases, especially in exon 2 , codons 12 (70-80\%) and 13 (15-20\%), while NRAS mutations occur in exons 2,3 and $4[20,21]$. BRAF also plays an essential role in the MAP-kinase (mitogen-activated protein kinase) pathway activation which contributes to cellular growth, proliferation, and differentiation, as well as to other vital cellular processes such as migration, apoptosis and cellular survival. Approximately $90 \%$ of $B R A F$ activating mutations occur in exon 15 [22, 23].

Concomitant RAS and BRAF mutations are rare: one study on mCRC patients indicated an incidence of $0.2 \%$ in a population of 6,633 patients, and in another 6,251-patient series the incidence was $0.064 \%[4,13]$. Thirty cases of concomitant $B R A F$ and KRAS mutations in mCRC patients have been described in the literature so far. However, the detection rate for these genetic lesions depends on the used technique. Real time PCR has been traditionally used for molecular characterization of CRC, but the modern NGS method has the potential to more precisely identify cu identify more precisely the incidence of concomitant RAS and BRAF mutations, which might be higher than previously estimated [16]. Given their rarity, it is not yet clear if tumors with concomitant RAS and BRAF mutations have a different tumor biology and natural history than those with individual RAS or BRAF mutations, and which of these two mutations play a more significant role in tumor invasion and aggressiveness [5]. Molecular profiling has demonstrated that dual RAS and BRAF mutant tumors have different genetic signatures suggesting that different signaling pathways are activated [24]. Moreover, it is still not known if RAS, BRAF or both are driver mutations [4]. It is believed that colorectal tumors originate in two clonal populations with the coexistence of two mutations at the same genetic level $[5,25]$. Several theories have attempted to explain the coexistence of these two mutations, but none is definitively proven. One possibility is that redundant oncogenic stimuli are activated. Another hypothesis states that these mutations exert a synergistic role in the stimulation of the disease progression $[26,27]$.

In their research, Oliveira et al. [27] were able to associate concurrent RAS and BRAF mutations with more advanced stages, observing an increased likelihood of lymph node invasion, distant metastases, and poor prognosis in these cases. However, other studies did not confirm this to be a consistent pattern. Because only 30 cases have been described in the literature up to the present time, we cannot conclude on clinical, pathological and molecular correlations. Relevant features of these 30 reported cases are summarized in Supplementary Table I [4-6, 12-19]. 
In these cases, we identified two molecular subtypes of BRAF-mutated mCRC: one with V600E mutation and the other with non-V600 mutation. Recent studies discuss these two phenotypes, and they show that they have indeed different clinical, molecular, histopathological and prognostic features [28]. Tumors with BRAF V600E mutations are most often located in the right colon and are prevalent in women and older patients (usually over 60 years), are poorly differentiated and are associated with mucinous histological type and MSIhigh [29]. Non-V600 mutations most often affect codons 594 and 596 and are less frequent (2.2\% of mCRC). These tumors occur more often in men, at younger ages, are welldifferentiated, located in the left colon, and are associated with $R A S$ mutation and rarely present MSI-high. This suggests that, at least in some cases, non-V600 BRAF mutations have a better prognosis than V600E $[28,30]$. At the same time, considering the existing molecular data, the following classification of $B R A F$ mutations has been proposed [30]: class 1 - BRAF $R A S$-independent mutations - with signaling as monomers; class 2 - BRAF RAS mutations - with independent signaling as dimers; class 3 - BRAF RAS dependent mutations - with impaired kinase activity.

Schirripa et al. [31] have demonstrated a correlation between these three classes and clinical characteristics. Class 3 was associated with left tumors, no lymph node and no peritoneal metastases (as opposed to class 1), while class 2 was similar to class 1 . Prognosis was different between subclasses - classes 1 and 2 had similar median overall survival (OS): 21 and 23 months, while class 3 and BRAF WT had a median OS of 44.5 months. This study confirms the significant survival differences between BRAF non-V600E mutations and BRAF V600E mutations, highlighting the less aggressive behavior of the former [31].

Data from literature are insufficient to guide choices of the appropriate type of systemic treatment in such cases [13]. Of the 30 patients, only 10 received chemotherapy with or without targeted therapy. Only one study included mCRC patients with concurrent KRAS/BRAF mutations who were treated exclusively by the surgical removal of the metastatic lesions, but the results were inconclusive.

The phase III TRIBE study included mCRC patients who received first-line treatment with either FOLFIRI plus bevacizumab or FOLFOXIRI plus bevacizumab. The results showed that patients with $\mathrm{mCRC}$ and BRAF mutation could benefit more from an intensive chemotherapy regimen. However, this study included a small number of patients $(n=28)$ and gains on progression-free survival (PFS) and OS, although present, were not statistically significant [32]. Recently, TRIBE 2 , a prospective randomized phase III trial included patients with treatment-naïve mCRC in two arms: in one arm subjects received FOLFOX and bevacizumab, followed by FOLFIRI at disease progression, and in the other arm FOLFOXIRI and bevacizumab, which was re-introduced at progression. The trial included 66 patients with BRAF mutations, 33 in each arm. The lack of benefit from the more intensive treatment observed in the BRAF mutated subgroup could be explained by the clinical heterogeneity of $B R A F$ mutant tumors or by a different comparator used in the TRIBE study (FOLFIRI) than in the TRIBE 2 study (FOLFOX). However, it seems that the effect of a more aggressive treatment in tumors with $B R A F$ mutation is different depending on the location of the primary tumor: subjects with tumors located on the right side seem to benefit most from the triplet, as the subgroup analyzes in this trial have shown [33].

Another therapeutic alternative in patients with $B R A F$ mutation is the inhibition of the RAF pathway. Various studies have shown modest activity of $B R A F$ inhibitors in monotherapy, but their use in combination with MEK inhibitor or EGFR inhibitors has yielded better results [34, 35]. A triple combination between encorafenib, cetuximab and a PIK3CA inhibitor, alpelisib, was explored in a phase I and in a phase II study but the results were not promising, and this strategy was abandoned [36, 37]. Other triple combinations between $B R A F$ inhibitors, MEK inhibitors, EGFR inhibitors, and/or chemotherapy were studied, and phase I and II studies created the rationale for a large phase III trial conducted by Kopetz et al. (BEACON study) $[38,39,40]$. Six hundred and sixtyfive mCRC patients with BRAF V600E mutation who had progressive disease after one or two lines of treatment were enrolled. The study had 3 arms: one arm received encorafenib, binimetinib and cetuximab, one arm encorafenib and cetuximab, and the control group cetuximab and irinotecan or cetuximab and FOLFIRI (at the investigator's choice). Patients who received the triplet had a better outcome with an OS of 9 months, versus those who received doublet ( 8.4 months) and those in the control group (5.4 months) $(\mathrm{p}<0.001)$ [40].

The TRIBE, TRIBE 2 and BEACON studies analyzed the efficacy of different treatments in the population of $B R A F$ V600E mutation patients, but there were no patients enrolled with concomitant RAS and BRAF mutations [32, 33, 40]. Although these treatments may be effective for patients with coexisting mutations, there is no data to support this idea, so new therapeutic strategies may be studied in the future for this very rare category of patients [11].

The role of $R A S$ and $B R A F$ mutations as prognostic factors has been evaluated by many researchers. $R A S$ mutations are considered a negative prognostic factor in multiple trials, but some data contradicts this result [42-44]. BRAF mutations are associated with a shorter OS (10-16 months) in mCRC patients. Multiple prospective, retrospective studies and a meta-analysis have confirmed these findings [42, 45-49]. Although some retrospective studies view concomitant mutations as a poor prognosis factor, more data on a more significant number of patients are required to adequately define the prognostic role of such a rare condition [13]. Compared to other cases in which survival was reported, the 23-month survival of our patient exceeded the 21.1-month median. Also, the survival of our patient was superior to the 18.5-month median OS reported for $B R A F$ mutated patients but was inferior to the 25.6-month survival of patients with RAS mutations [50].

Our case shares many features with those previously reported, but very few with the $B R A F$ V600E mutant patients: clinical (male, 51 years old), pathological (rectal adenocarcinoma) and molecular (KRAS G12D mutation and $B R A F$ V600E mutation). On the other hand, the patient had similar survival with the average mentioned in the literature: 23 months following diagnosis. This could suggest that concomitant KRAS and BRAF mutations may not necessarily 
be a poorer prognostic factor than $B R A F$ mutations. There is still a plethora of other factors that could have impacted the prognosis of our patient: the rectal localization of the primary tumor, the fact that the patient was not tested by NGS for other mutations, the genetic heterogeneity of CRC tumors, or the consensus molecular subtype [5].

\section{CONCLUSIONS}

Prospective or retrospective cohort studies on a large number of patients are necessary in order to adequately assess the clinical, pathological and molecular characteristics of patients with mCRC and concomitant KRAS and BRAF mutations. Such studies would provide useful insights informing appropriate treatment choices in accordance with the patient profile and prognosis data.

Conflicts of interest: None to declare.

Authors' contributions: V.A.A. conceived and designed the article. M.V.M., T.A.S. data analysis and drafted the manuscript. T.A.S., A.V.I. searched in the literature. A.E.C., B.G., L.M., R.C supervised and revised the manuscript. All authors critically revised the manuscript, approved the final version to be published, and agree to be accountable for all aspects of the work.

Supplementary material: To access the supplementary material visit the online version of the $J$ Gastrointestin Liver Dis at http://dx.doi. org/10.15403/jgld-1003

\section{REFERENCES}

1. Parkin DM, Bray F, Ferlay J, Pisani P. Global cancer statistics 2002. CA Cancer J Clin 2002;55:74-108. doi:10.3322/canjclin.55.2.74

2. Wolpin BM, Mayer RJ. Systemic treatment of colorectal cancer. Gastroenterology 2008;134:1296-1310. doi:10.1053/j.gastro.2008.02.098

3. Kelly H, Goldberg RM. Systemic therapy for metastatic colorectal cancer: current options, current evidence. J Clin Oncol 2005;23:45534560. doi:10.1200/JCO.2005.17.749

4. Sahin IH, Kazmi SM, Yorio JT, Bhadkamkar NA, Kee BK, Garrett CR. Rare though not mutually exclusive: A report of three cases of concomitant KRAS and BRAF mutation and a review of the literature. J Cancer 2013;4:320-322. doi:10.7150/jca.3619

5. Larki P, Gharib E, Yaghoob Taleghani M, Khorshidi F, Nazemalhosseini-Mojarad E, Asadzadeh Aghdaei H. Coexistence of KRAS and BRAF mutations in colorectal cancer: a case report supporting the concept of tumoral heterogeneity. Cell J 2017;19(suppl 1):113-117. doi:10.22074/cellj.2017.5123

6. Vittal A, Middinti A, Kasi Loknath Kumar A. Are all mutations the same? A rare case report of coexisting mutually exclusive KRAS and BRAF mutations in a patient with metastatic colon adenocarcinoma. Case Rep Oncol Med 2017;2017:2321052. doi:10.1155/2017/2321052

7. Douillard JY, Siena S, Cassidy J, et al. Randomized, phase III trial of panitumumab with infusional fluorouracil, leucovorin, and oxaliplatin (FOLFOX4) versus FOLFOX4 alone as first-line treatment in patients with previously untreated metastatic colorectal cancer: the PRIME study. J Clin Oncol 2010;28:4697-4705. doi:10.1200/JCO.2009.27.4860
8. Douillard JY, Oliner KS, Siena S, et al. Panitumumab-FOLFOX4 treatment and RAS mutations in colorectal cancer. N Engl J Med 2013;369:1023-1034. doi:10.1056/NEJMoa1305275

9. Ciardiello F, Lenz HJ, Köhne CH, et al. Treatment outcome according to tumor RAS mutation status in CRYSTAL study patients with metastatic colorectal cancer (mCRC) randomized to FOLFIRI with/ without cetuximab. J Clin Oncol 2014;32(suppl):3506. doi:10.1200/ jco.2014.32.15_suppl.3506

10. Bokemeyer C, Köhne CH, Ciardiello F, et al. FOLFOX4 plus cetuximab treatment and RAS mutations in colorectal cancer. Eur J Cancer 2015;51:1243-1252. doi:10.1016/j.ejca.2015.04.007

11. Sanz-Garcia E, Argiles G, Elez E, Tabernero J. BRAF mutant colorectal cancer: prognosis, treatment, and new perspectives. Ann Oncol 2017;28:2648-2657. doi:10.1093/annonc/mdx401

12. Guglielmini PF, Rossi M, Grosso M, et al. Double KRAS and BRAF mutations in colorectal cancer in a single oncologic department series. J Clin Oncol 2017;15(suppl):e14657. doi:10.1200/jco.2013.31.15_suppl. e14657

13. Deshwar A, Margonis GA, Andreatos N, et al. Double KRAS and BRAF mutations in surgically treated colorectal cancer liver metastases: An international multi-institutional case series. Anticancer Res 2018;38(5):2891-2895. doi:10.21873/anticanres.12535

14. Zheng G, Tseng GH, Haley L, et al. Clinical validation of coexisting driver mutation in colorectal cancers. Hum Pathol 2019;86:12-20. doi:10.1016/j.humpath.2018.11.014

15. Vittal A, Sharma D, Samanta I, Kasi A. Rare case of triple mutant (KRAS + NRAS + BRAF) metastatic colon adenocarcinoma. BMJ Case Rep 2019;12:e221816. doi:10.1136/bcr-2017-221816

16. Midthun L, Shaheen S, Deisch J, Senthil M, Tsai J, Hsueh CT. Concomitant KRAS and BRAF mutations in colorectal cancer. J Gastrointest Oncol 2019;10:577-581. doi:10.21037/jgo.2019.01.10

17. Ates $\mathrm{O}$, Yalcin S. Concomitant RAS and BRAF mutation in colorectal cancer. A report of 7 cases. Indian J Cancer 2019;56:176-179. doi:10.4103/ijc.IJC_430_18

18. Isnaldi E, Garuti A, Cirmena G, et al. Clinico-pathological associations and concomitant mutations of the RAS/RAF pathway in metastatic colorectal cancer. J Transl Med 2019;17:137. doi:10.1186/s12967-019. 1879-2

19. Vidal J, Bellosillo B, Santos Vivas C, et al. Ultra-selection of metastatic colorectal cancer patients using next-generation sequencing to improve clinical efficacy of anti-EGFR therapy. Ann Oncol 2019;30:439-446. doi:10.1093/annonc/mdz005

20. Afrăsânie VA, Marinca MV, Alexa-Stratulat T, et al. KRAS, NRAS, BRAF, HER2 and microsatellite instability in metastatic colorectal cancer practical implications for the clinician. Radiol Oncol 2019;53:265-274. doi:10.2478/raon-2019-0033

21. Gong J, Cho M, Sy M, Salgia R, Fakih M. Molecular profiling of metastatic colorectal tumors using next-generation sequencing: a singleinstitution experience. Oncotarget 2017;8:42198-42213. doi:10.18632/ oncotarget. 15030

22. Wan PT, Garnett MJ, Roe SM, et al. Mechanism of activation of the RAF-ERK signaling pathway by oncogenic mutations of BRAF. Cell 2004;116:855-867. doi:10.1016/S0092-8674(04)00215-6

23. Davies H, Bignell GR, Cox C, et al. Mutations of the BRAF gene in human cancer. Nature 2002;417:949-954. doi:10.1038/nature00766

24. Seth R, Crook S, Ibrahem S, Fadhil W, Jackson D, Ilyas M. Concomitant mutations and splice variants in KRAS and BRAF demonstrate complex perturbation of the RAS/RAF signalling pathway in advanced colorectal cancer. Gut 2009;58:1234-1241. doi:10.1136/gut.2008.159137 
25. De Roock W, Piessevaux H, De Schutter J, et al. KRAS wild-type state predicts survival and is associated to early radiological response in metastatic colorectal cancer treated with cetuximab. Ann Oncol 2008;19:508-515. doi:10.1093/annonc/mdm496

26. Morkel M, Riemer P, Blaker H, Sers C. Similar but different: distinct roles for KRAS and BRAF oncogenes in colorectal cancer development and therapy resistance. Oncotarget 2015;6:20785-20800. doi:10.3390/ jcm 8010111

27. Oliveira C, Velho S, Moutinho C, et al. KRAS and BRAF oncogenic mutations in MSS colorectal carcinoma progression. Oncogene 2007;26:158-163. doi:10.1038/sj.onc.1209758

28. Caputo F, Santini C, Bardasi C, et al. BRAF-mutated colorectal cancer clinical and molecular insights. Int J Mol Sci 2019;20(21):5369. doi:10.3390/ijms20215369

29. van Cutsem E, Dekervel J. Not all BRAF-mutant metastatic colorectal cancers are identical: distinct clinical consequences of non-V600 BRAF mutations. J Clin Oncol 2017;35:2598-2599. doi:10.1200/ JCO.2017.72.7057

30. Yao Z, Yaeger R, Rodrik-Outmezguine VS, et al. Tumours with class 3 BRAF mutants are sensitive to inhibitions of activated RAS. Nature 2017;548:234-238. doi:10.1038/nature23291

31. Schirripa M, Biason P, Lonardi S, et al. Class 1, 2 and 3 BRAF-mutated metastatic colorectal cancer: A detailed clinical, pathological and molecular characterization. Clin Cancer Res 2019;25:3954-3961. doi:10.1158/1078-0432.CCR-19-0311

32. Cremolini C, Loupakis F, Antoniotti C, et al. FOLFOXIRI plus bevacizumab versus FOLFIRI plus bevacizumab as first-line treatment of patients with metastatic colorectal cancer: updated overall survival and molecular subgroup analyses of the open-label, phase 3 TRIBE study. Lancet Oncol 2015;16:1306-1315. doi:10.1016/S14702045(15)00122-9

33. Cremolini C, Antoniotti C, Rossini D, et al. Upfront FOLFOXIRI plus bevacizumab and reintroduction after progression versus mFOLFOX6 plus bevacizumab followed by FOLFIRI plus bevacizumab in the treatment of patients with metastatic colorectal cancer (TRIBE2): a multicentre, open-label, phase 3, randomised, controlled trial. Lancet Oncol 2020;21:497-507. doi:10.1016/S1470-2045(19)30862-9

34. Taieb J, Lapeyre-Prost A, Laurent Puig P, Zaanan A. Exploring the best treatment options for BRAF-mutant metastatic colon cancer. Br J Cancer 2019;121:434-442. doi:10.1038/s41416-019-0526-2

35. Ducreux M, Chaseddine A, Laurent-Puig P, et al. Molecular targeted therapy of BRAF-mutant colorectal cancer. Ther Adv Med Oncol 2019;11:1758835919856494. doi:10.1177/1758835919856494

36. van Geel R, Tabernero J, Elez E, et al. A Phase Ib Dose-Escalation Study of Encorafenib and Cetuximab with or without Alpelisib in Metastatic BRAF-Mutant Colorectal Cancer. Cancer Discov 2017;7(6):610-619. doi:10.1158/2159-8290

37. Tabernero J, van Geel R, Guren TK, et al. Phase 2 results: encorafenib (ENCO) and cetuximab (CETUX) with or without alpelisib (ALP) in patients with advanced BRAF-mutant colorectal cancer (BRAFm CRC). J Clin Oncol 2016;34:3544. doi:10.1200/JCO.2016.34.15_suppl.3544
38. Corcoran RB, André T, Atreya CE, et al. Combined BRAF, EGFR, and MEK Inhibition in Patients with $B R A F^{\mathrm{V} 600 \mathrm{E}}$ Mutant Colorectal Cancer. Cancer Discov 2018;8:428-443. doi:10.1158/2159-8290.CD$17-1226$

39. Hong DS, Morris VK, El Osta B, et al. Phase IB Study of vemurafenib in combination with irinotecan and cetuximab in patients with metastatic colorectal cancer with BRAFV600E mutation. Cancer Discov 2016;6:1352-1365. doi:10.1158/2159-8290.CD-16-0050

40. Kopetz S, Grothey A, Yaeger R, et al. Encorafenib, binimetinib, and cetuximab in BRAF V600E-mutated colorectal cancer. N Engl J Med 2019;381:1632-1643. doi:10.1056/NEJMoa1908075

41. Tran B, Kopetz S, Tie J, et al. Impact of BRAF mutation and microsatellite instability on the pattern of metastatic spread and prognosis in metastatic colorectal cancer. Cancer 2011;117:4623-4632. doi:10.1002/ cncr. 26086

42. Garcia-Carbonero N, Martinez-Useros J, Li W, et al. KRAS and BRAF mutations as prognostic and predictive biomarkers for standard chemotherapy response in metastatic colorectal cancer: A single institutional study. Cells 2020;9:E219. doi:10.3390/cells9010219

43. Schirripa M, Cremolini C, Loupakis F, et al. Role of NRAS mutations as prognostic and predictive markers in metastatic colorectal cancer. Int J Cancer 2015;136:83-90. doi:10.1002/ijc.28955

44. De Roock W, Claes B, Bernasconi D, et al. Effects of KRAS, BRAF, NRAS and PIK3CA mutations on the efficacy of cetuximab plus chemotherapy in chemotherapy-refractory metastatic colorectal cancer: a retrospective consortium analysis. Lancet Oncol 2010;11:753-762. doi:10.1016/ S1470-2045(10)70130-3

45. Maughan TS, Adams RA, Smith CG, et al. Addition of cetuximab to oxaliplatin-based first-line combination chemotherapy for treatment of advanced colorectal cancer: results of the randomised phase 3 MRC COIN trial. Lancet 2011;377:2103-2114. doi:10.1016/S01406736(11)60613-2

46. Bokemeyer C, Bondarenko I, Hartmann JT, et al. Efficacy according to biomarker status of cetuximab plus FOLFOX-4 as first-line treatment for metastatic colorectal cancer: the OPUS study. Ann Oncol 2011;22:15351546. doi:10.1093/annonc/mdq632

47. Douillard JY, Siena S, Cassidy J, et al. Final results from PRIME: randomized phase III study of panitumumab with FOLFOX4 for first-line treatment of metastatic colorectal cancer. Ann Oncol 2014;25:1346-1355. doi:10.1093/annonc/mdu141

48. Van Cutsem E, Lenz HJ, Köhne CH, et al. Fluorouracil, leucovorin, and irinotecan plus cetuximab treatment and RAS mutations in colorectal cancer. J Clin Oncol 2015;33:692-700. doi:10.1200/JCO.2014.59.4812

49. Pietrantonio F, Petrelli F, Coinu A, et al. Predictive role of BRAF mutations in patients with advanced colorectal cancer receiving cetuximab and panitumumab: a meta-analysis. Eur J Cancer 2015;51:587-594. doi:10.1016/j.ejca.2015.01.054

50. Kayhanian H, Goode E, Sclafani F, et al. Treatment and survival outcome of BRAF-mutated metastatic colorectal cancer: a retrospective matched case-control study. Clin Colorectal Cancer 2018;17:e69-e76. doi:10.1016/j.clcc.2017.10.006 